\title{
Trastuzumab-Induced Cardiomyopathy and Intermittent Left Bundle Branch Block
}

\author{
Hassan Tahir a, b, Nikky Bardia ${ }^{\mathrm{a}}$, Kulwant Bath ${ }^{\mathrm{a}}$, Yasir Ahmed ${ }^{\mathrm{a}}$, Muhammad Rafique ${ }^{\mathrm{a}}$, \\ Bassam Omara ${ }^{\text {a }}$ Christopher Malozzi ${ }^{\text {a }}$
}

\begin{abstract}
Trastuzumab-induced cardiomyopathy is a known complication of its use in breast cancer treatment, but it remains mostly asymptomatic and often reversible. Non-myopathic cardiac complications have been rarely reported with trastuzumab. These include left and right bundle branch block, arrhythmias and sinus node dysfunction. We report a case of a 52-year-old female breast cancer patient with trastuzumabinduced asymptomatic intermittent left bundle branch block recurring nearly a year after the initial diagnosis and resolution of trastuzumabrelated cardiomyopathy.
\end{abstract}

Keywords: Trastuzumab; Transient left bundle branch block; Nonmyopathic cardiac complications

\section{Introduction}

Trastuzumab is a humanized monoclonal antibody directed against human epidermal growth factor receptor 2 (HER2), which is expressed in about $20-25 \%$ of breast cancers and promotes growth of cancer cells [1]. This targeted immunotherapy is approved for the treatment of HER2-positive breast cancer [2]. Trastuzumab exhibits its anticancer attributes by binding the extracellular domain IV of HER2, and thereby directly modulating pro-survival and proliferative signaling cascades, including phosphatidylinositol 3-kinase (PI3K) and mitogenactivated protein kinase (MAPK) pathways [3, 4].

Breast cancer is one of the most common cancers among American women, affecting one in eight (12\%) females [5]. Clinically, trastuzumab has been shown to be highly efficacious as a single agent for the treatment of breast cancer or in conjunction with anthracyclines. However, it has been associated with numerous cardiotoxic effects. The most common cardiac complication of trastuzumab is cardiomyopathy

Manuscript submitted May 24, 2019, accepted June 7, 2019

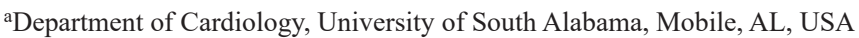
${ }^{b}$ Corresponding Author: Hassan Tahir, Department of Cardiology, University of South Alabama, 10th Floor, Ste B, Mobile, AL 36617, USA.

Email: htahir@health.southalabama.edu

doi: https://doi.org/10.14740/cr888
[6]. However, more non-myopathic cardiac complications are being reported due to a significant rise in the use of trastuzumab among breast cancer patients [6]. We report a case of a 52-year-old Caucasian woman with HER2-positive breast cancer who experienced recurrent, intermittent, asymptomatic LBBB nearly a year after trastuzumab use causing reversible cardiomyopathy with systolic dysfunction.

\section{Case Report}

A 52-year-old Caucasian woman with biopsy proven stage IIB, grade 3, HER2-positive, invasive carcinoma of the left breast was referred to cardiology for evaluation of chemotherapy-related cardiotoxicity. Her neo-adjuvant chemotherapeutic regimen included dose-dense adriamycin and cyclophosphamide (DDAC) along with paclitaxel, trastuzumab and pertuzumab. She had no prior history of cardiovascular disease. Her risk factors were tobacco use and family history of coronary artery disease (CAD).

The patient's initial multigated acquisition (MUGA) scan prior to beginning treatment showed left ventricular ejection fraction (LVEF) of $80.9 \%$. Upon completion of neo-adjuvant therapy, she underwent left mastectomy. Radiation therapy was discussed but had not been started. Following surgery, she restarted trastuzumab. A follow-up MUGA scan showed a decline in LVEF to $30.2 \%$. Subsequent transthoracic echocardiogram (TTE) confirmed severely reduced LVEF. No significant valvular regurgitant or stenotic lesions were noted. Electrocardiogram (ECG) obtained at the referral visit revealed normal sinus rhythm (NSR) and an LBBB with a QRS duration of $124 \mathrm{~ms}$ (Fig. 1). This was new compared to a prior ECG from 3 years earlier (Fig. 2). She denied symptoms of shortness of breath, orthopnea, paroxysmal nocturnal dyspnea, bendopnea or leg swelling. She reported the ability to walk briskly without limitations or symptoms. The patient was started on beta-blocker (BB) and angiotensin-converting enzyme inhibitor (ACE-I) therapy. Trastuzumab therapy was discontinued. Over the next 8 months, LVEF was reassessed with TTE and eventually returned to normal, $50-55 \%$. The patient remained asymptomatic during this time but was not able to resume trastuzumab.

A year after the patient's first cardiology encounter and approximately 5 months after recovery of systolic function, the patient required surgical repair of a complication from her initial mastectomy. This was scheduled to be performed at a 


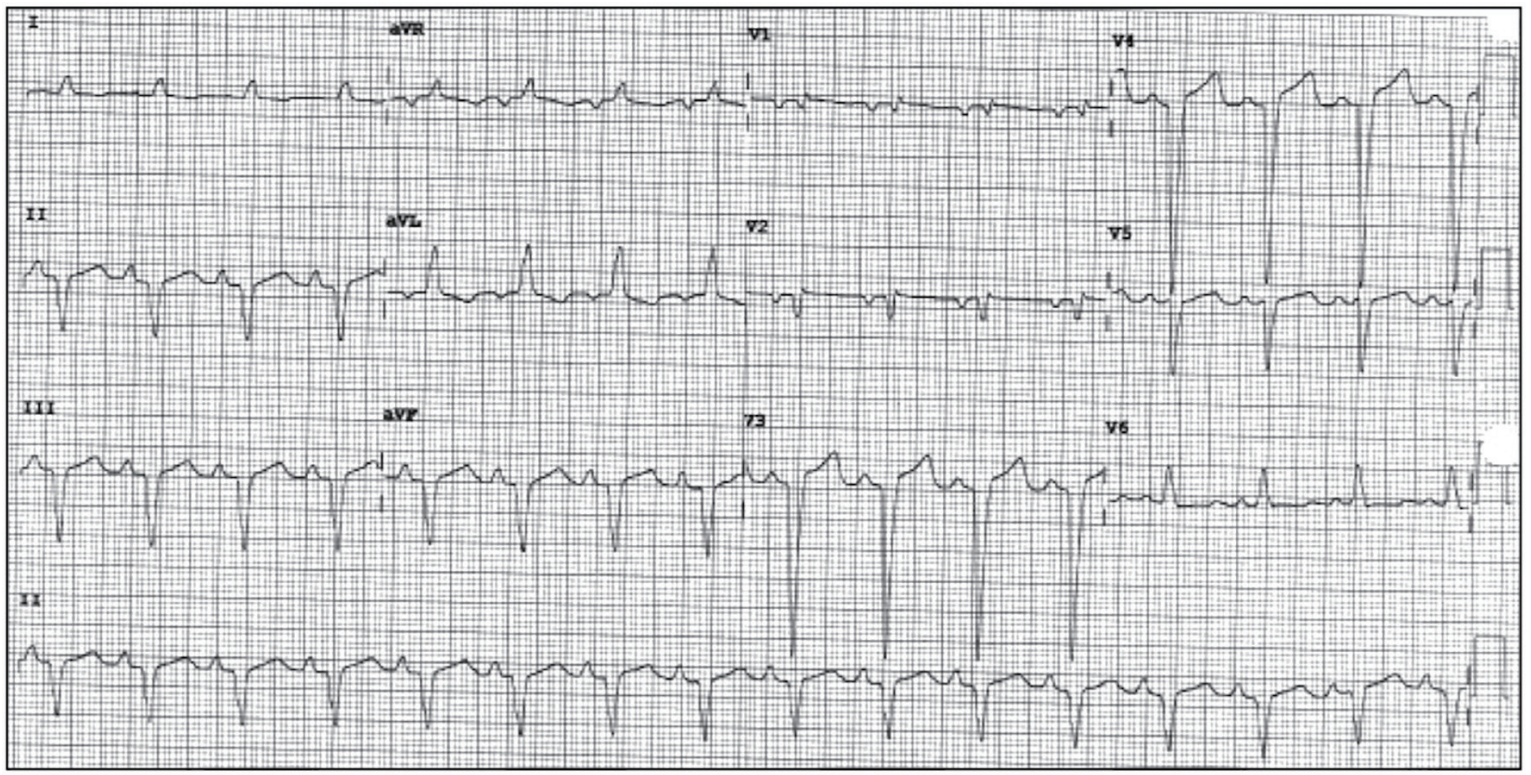

Figure 1. Presenting ECG to cardiology clinic with NSR and newly recognized LBBB. ECG: electrocardiogram; NSR: normal sinus rhythm; LBBB: left bundle branch block.

neighboring hospital. Preoperative ECG revealed an LBBB not previously documented at that facility (Fig. 3). The procedure was cancelled, and the patient referred back to cardiology for preoperative risk assessment. ECG at time of the visit revealed NSR and resolution of the LBBB noted 1 week prior (Fig. 4). However, new ST-T wave changes were present concerning for ischemia. The patient was referred for limited echocardiography and exercise stress myocardial perfusion imaging. Exercise stress testing was considered given the absence of LBBB on ECG in clinic and the concern for intermittent LBBB, possibly rate- or exercise-related. Limited echocardiogram revealed normal EF, 50-55\%. During exercise, the patient de- veloped LBBB when the heart rate (HR) reached 120 beat per minute (bpm) and resolved during rest when the HR returned to below $90 \mathrm{bpm}$ (Fig. 5). The patient had persistent anterior $\mathrm{T}$-wave inversions. The stress test was converted to a pharmacologic stress imaging exam. The myocardial perfusion scans revealed a small area of reversible ischemia in the anteroseptal wall vs. LBBB artifact (Fig. 6). The patient was referred to coronary angiography for definitive diagnosis of CAD. Left heart catheterization (LHC) revealed an LVEF of $60 \%$ and a right dominant system with angiographically normal arteries (Fig. 7). The patient underwent her surgical procedure without complications and continues on BB and ACE-I therapy along

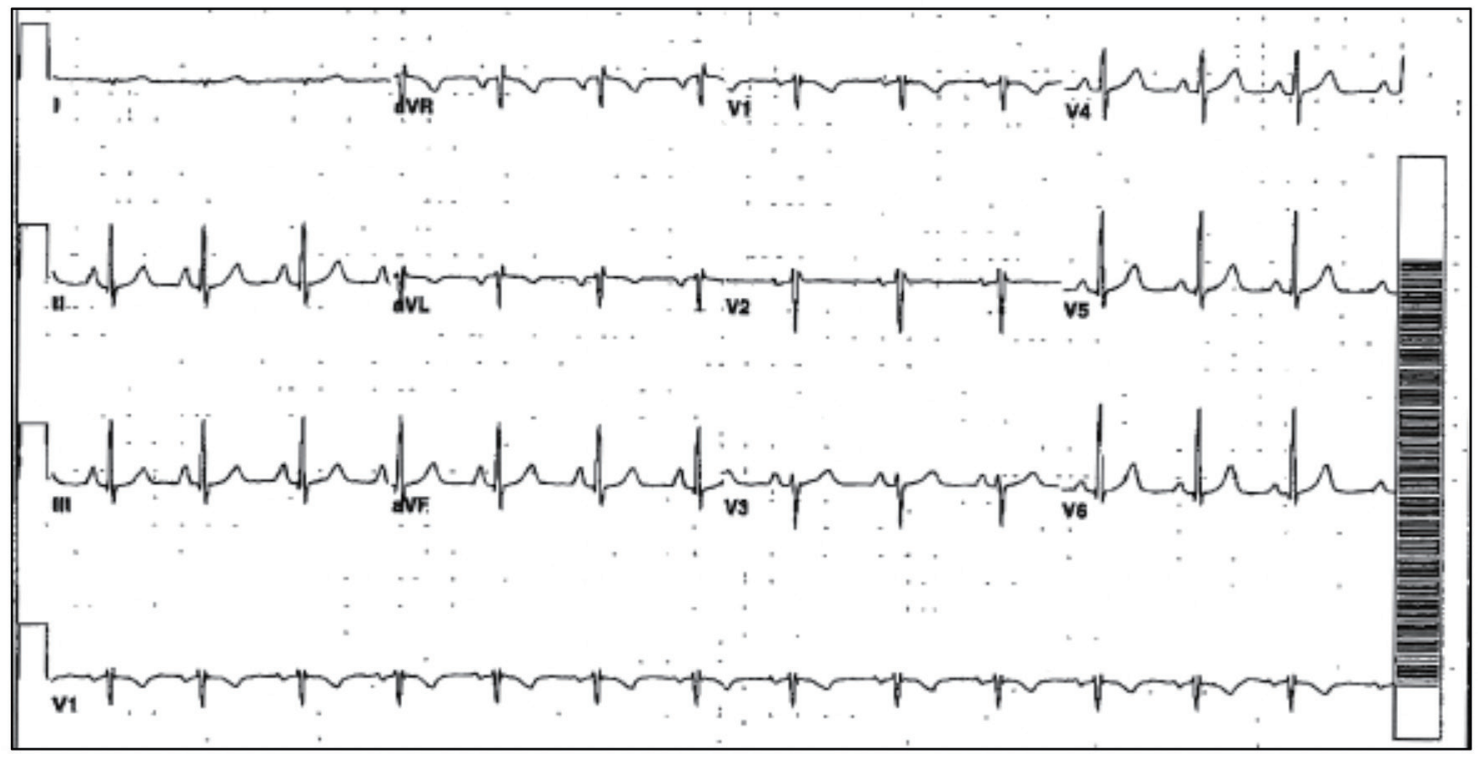

Figure 2. Prior ECG with NSR and normal conduction. ECG: electrocardiogram; NSR: normal sinus rhythm. 


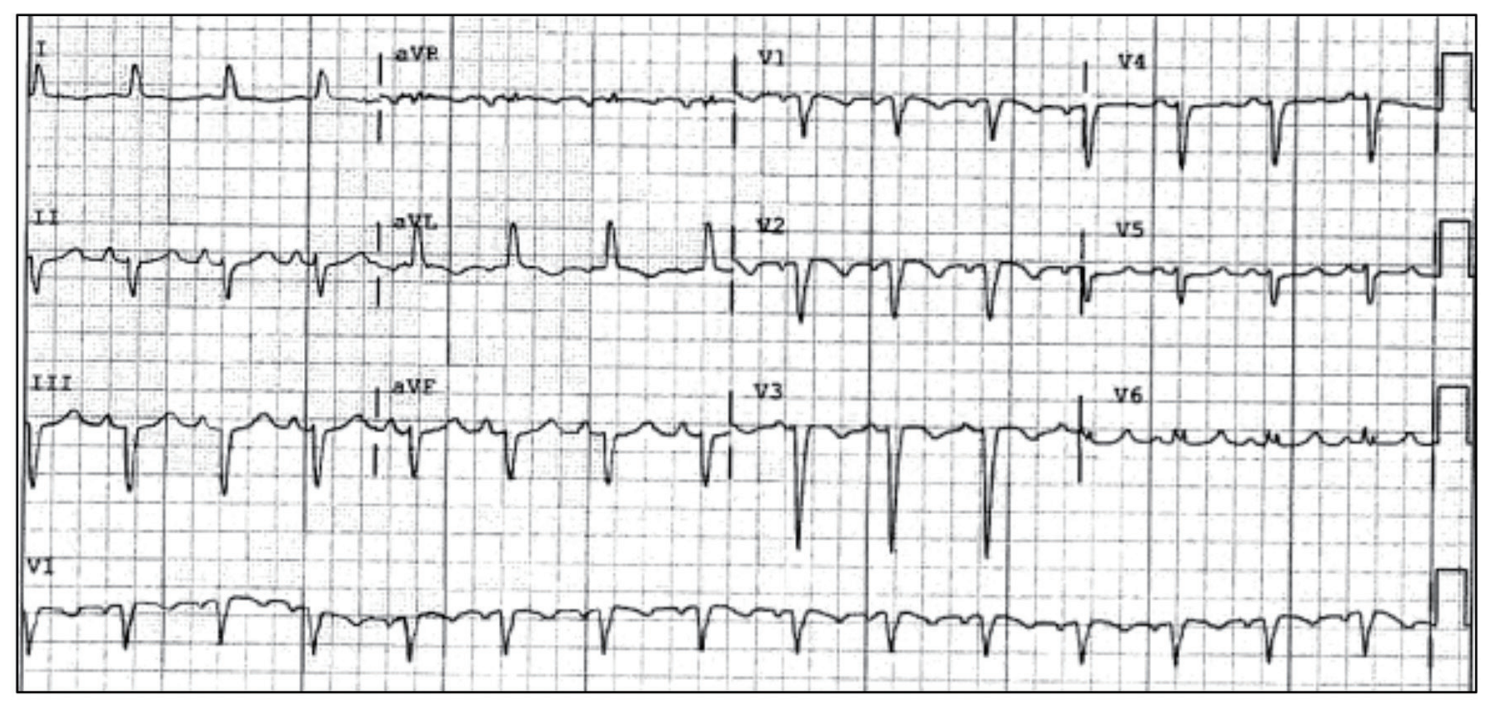

Figure 3. Preoperative ECG with recurrent LBBB. ECG: electrocardiogram; LBBB: left bundle branch block.

with cardiovascular risk factor modification.

\section{Discussion}

Trastuzumab is likely the most clinically important biologic agent developed for the treatment of HER2-positive breast cancer to date. Various clinical trials have shown reduction of cancer recurrence, increased response rates, increased progression-free survival and improvement in overall survival with addition of trastuzumab to standard chemotherapy [3, 7]. Because of this evidence, in 1998, The United States Food and Drug Administration approved trastuzumab for metastatic breast cancer treatment, but in 2006, its use was expanded for treatment of adjuvant HER2-positive breast cancer as well
[7]. However, left ventricular systolic dysfunction from trastuzumab is a known potential consequence of treatment. Other potential adverse cardiac side effects have been described as well. The underlying mechanism leading to cardiac problems is still unclear. Some proposed mechanisms are defective HER2 signaling, immune-mediated destruction of cardiomyocytes and inhibition of the neuregulin-ErbB system $[2,8]$.

The most common cardiotoxicity associated with trastuzumab is a decline in LVEF. Most patients remain asymptomatic and therefore systolic dysfunction is found on routine surveillance echocardiography done specifically to monitor for this. Clinical, symptomatic heart failure is less common. Regardless, systolic dysfunction is often reversible and responds to conventional heart failure therapy [9]. The risk of cardiac dysfunction is significantly higher when trastuzumab is used

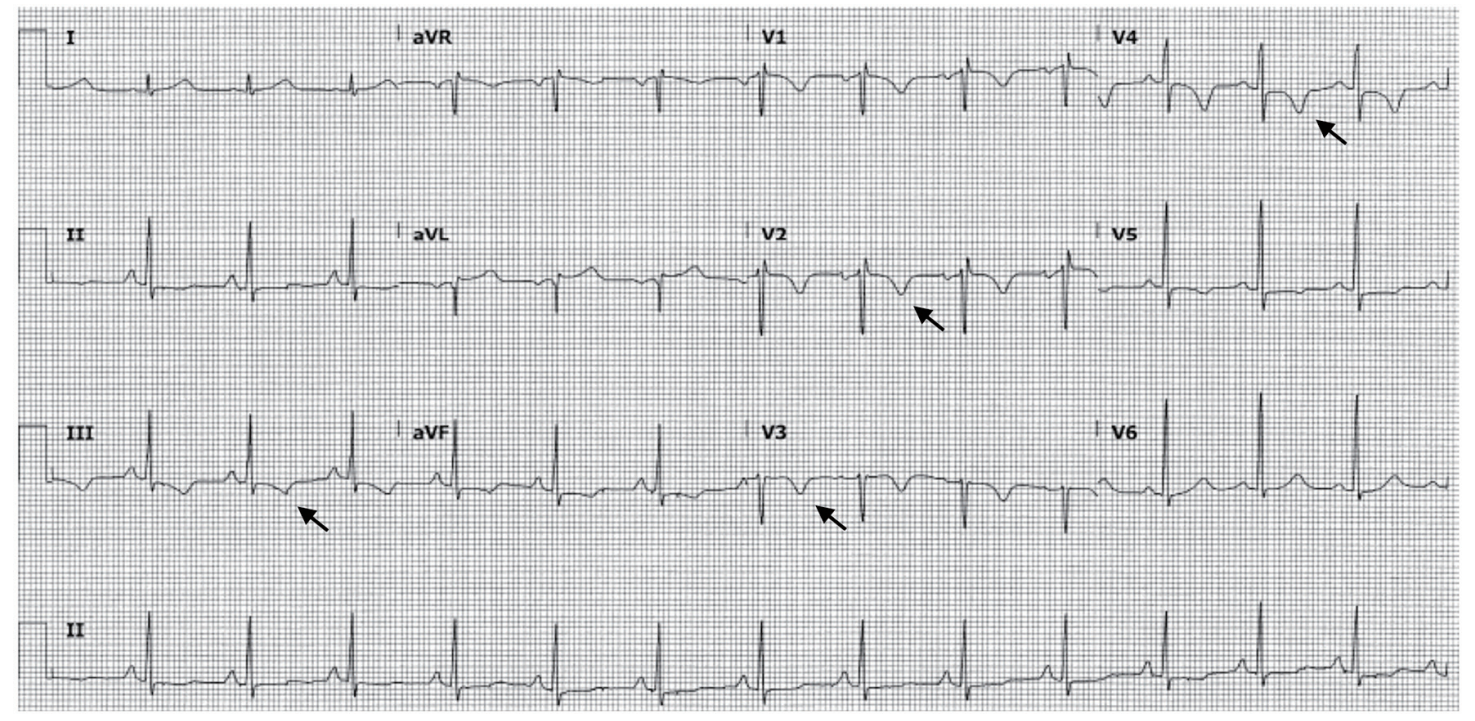

Figure 4. ECG obtained upon patient's return to cardiology clinic for preoperative risk assessment. This tracing shows the absence of LBBB and new T-wave inversions in multiple leads (arrows). ECG: electrocardiogram; LBBB: left bundle branch block. 

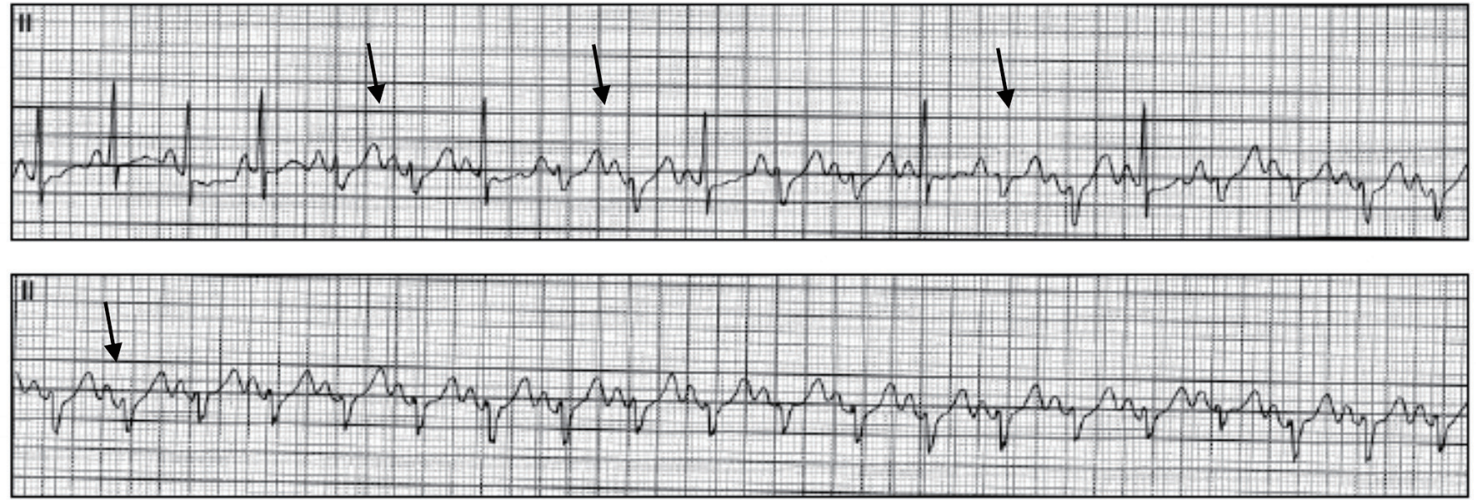

Figure 5. Representative ECG tracings during exercise stress revealing the development of intermittent LBBB (arrows). ECG: electrocardiogram; LBBB: left bundle branch block.

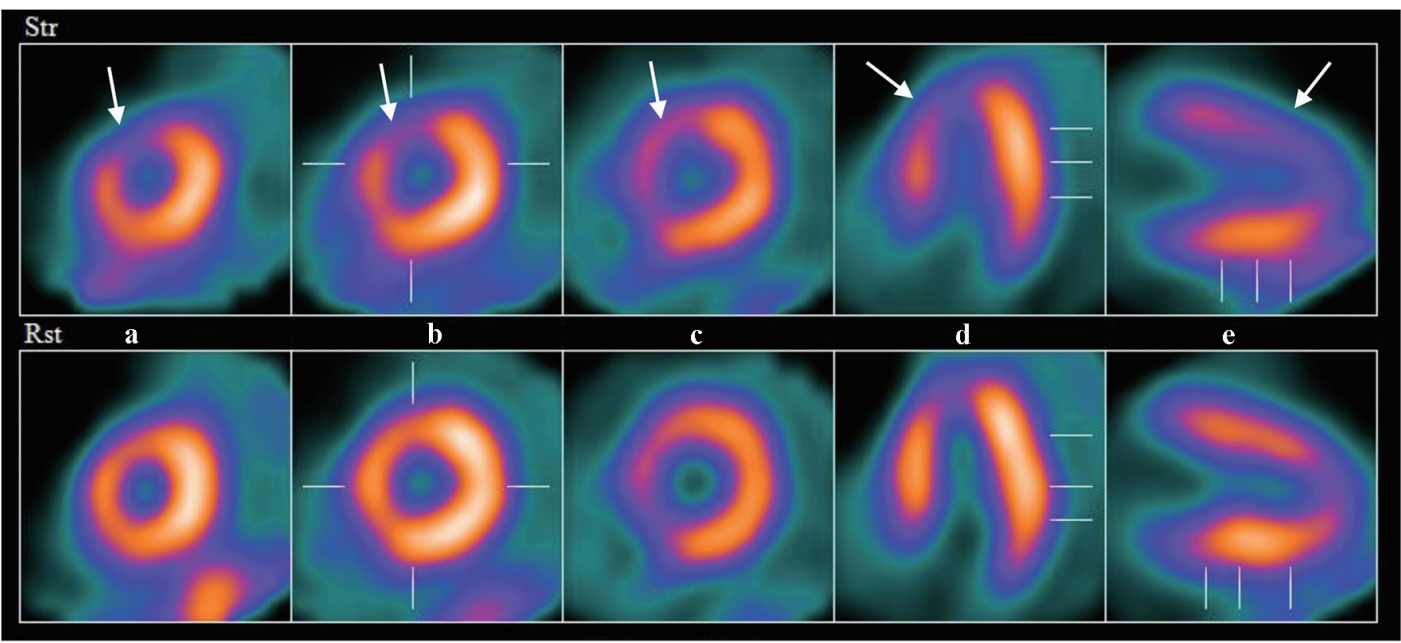

Figure 6. Example images from nuclear perfusion testing suggesting anteroseptal ischemia (arrows). The top row of images are stress images and the bottom are rest images (displayed left to right: short axis apex to base (a-c), horizontal long axis (d) and vertical long axis (e)).
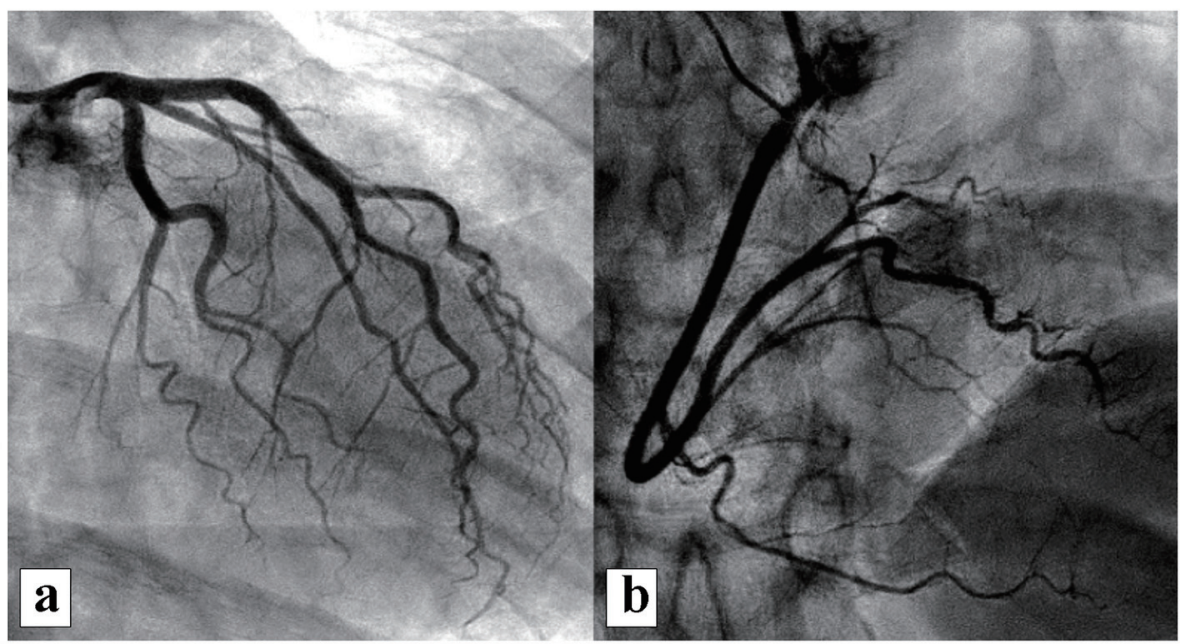

Figure 7. Representative images from coronary angiography revealing no significant CAD: (a) left coronary system; (b) right coronary system. CAD: coronary artery disease. 
Table 1. Non-Myopathic Cardiac Complications Associated With Trastuzumab [6, 13-17]

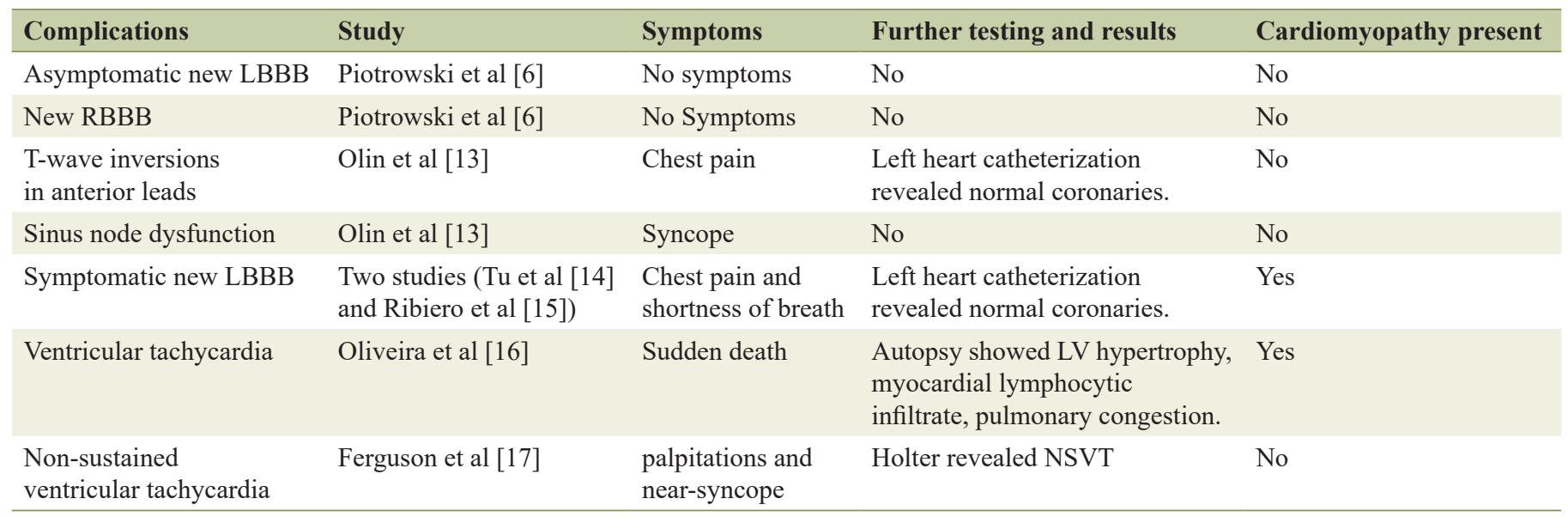

LBBB: left bundle branch block; RBBB: right bundle branch block; LV: left ventricular; NSVT: non-sustained ventricular tachycardia.

in combination with other antineoplastic agents, such as cyclophosphamide and anthracyclines [10]. It has been reported that cardiotoxicity occurs in approximately $4 \%$ of patients receiving trastuzumab monotherapy and $27 \%$ of patients receiving it along with anthracycline and cyclophosphamide [9]. This side effect has been well established, and guidelines exist regarding echocardiographic monitoring and whether trastuzumab should be continued or withheld, temporarily or permanently [11].

On the other hand, there are also less well-recognized, nonmyopathic, cardiac complications that have been described. These are rarely reported and are mostly asymptomatic as well $[12,13]$. Table $1[6,13-17]$ lists the non-myopathic cardiac complications of trastuzumab reported in literature. Piotrowski and colleagues followed 253 women with early HER2-positive breast cancer treated with trastuzumab [6]. A cardiac complication developed in 52 patients, out of which only one had permanent asymptomatic LBBB which occurred 3 months after starting therapy. Trastuzumab was discontinued in this particular patient; however, the conduction problem persisted. Of note, LBBB in this case was not associated with cardiomyopathy. Asymptomatic right bundle branch block (RBBB) also occurred in two patients but therapy was not discontinued [6]. There are two reported cases of new LBBB in patients who presented with chest pain mimicking acute coronary syndrome. Both patients underwent LHC and neither had significant CAD $[14,15]$. LBBB was persistent in one case [14] and transient in the other [15]. Both of these cases were also associated with reduced LVEF and were treated with guideline-directed medical therapy. Right ventricular failure has also been reported with trastuzumab use [18].

This particular case is unique in that the patient exhibited two, possibly three, cardiac side effects of trastuzumab. She initially presented as newly diagnosed, asymptomatic cardiomyopathy and LBBB. Both of these effects eventually resolved with cessation of trastuzumab and routine heart failure therapy. However, despite a normal LVEF, the patient had recurrent but intermittent LBBB. When normal conduction was present, she also exhibited anterior T-wave inversion, also a previously described cardiac electrical complication [12]. CAD was excluded by LHC as a cause for all the ECG abnormalities.

This report reviews the many possible cardiac complications of trastuzumab therapy. It also highlights the less recognized potential non-myopathic cardiac effects. More importantly, the long-term follow-up in this patient emphasizes that trastuzumab cardiotoxicity can occur at any time during or after treatment and manifest itself in many different ways. Therefore, a patient exposed to trastuzumab must have diligent cardiac monitoring both during and after treatment, especially since many of these toxicities are asymptomatic. However, at this time there are no guidelines available for short-term or long-term monitoring of non-myopathic side effects as there are with surveillance for cardiomyopathy. Also, decision regarding discontinuation of trastuzumab after asymptomatic, non-myopathic cardiac complications is still a topic of debate and an opportunity for future research.

\section{Conclusion}

Trastuzumab has been associated with an increased risk of asymptomatic decrease in ejection fraction, and, in a small number of patients, symptomatic congestive heart failure. In both situations, the cardiotoxicity is mostly responsive to medical management. This risk, however, is compounded when used in combination with anthracyclines as part of the chemotherapy regimen. Non-myopathic cardiac complications of trastuzumab are either rare or underreported. Increased use of this medication has led increased identification of additional cardiac side effects. The natural course of these rare complications is unknown. More research is needed to study the association of these complications with trastuzumab use and its impact on breast cancer treatment.

\section{Acknowledgments}

None. 


\section{Financial Disclosure}

None.

\section{Conflict of Interest}

None.

\section{Informed Consent}

Consent was taken from patient.

\section{Author Contributions}

All authors participated sufficiently in the intellectual content, review of literature and the analysis of data. Each author has reviewed the final version of the manuscript and approves it for publication.

\section{References}

1. Toikkanen S, Helin H, Isola J, Joensuu H. Prognostic significance of HER-2 oncoprotein expression in breast cancer: a 30-year follow-up. J Clin Oncol. 1992;10(7):10441048 .

2. Hudis CA. Trastuzumab - mechanism of action and use in clinical practice. N Engl J Med. 2007;357(1):39-51.

3. Slamon DJ, Leyland-Jones B, Shak S, Fuchs H, Paton V, Bajamonde A, Fleming T, et al. Use of chemotherapy plus a monoclonal antibody against HER2 for metastatic breast cancer that overexpresses HER2. N Engl J Med. 2001;344(11):783-792.

4. Wu WJ, Dokmanovic M. Trastuzumab. In: Schwab M., editor. Encyclopedia of Cancer. Berlin, Heidelberg, Germany: Springer Reference; Springer-Ver- lag; 2009. p. 3048-3049.

5. Miller KD, Siegel RL, Lin CC, Mariotto AB, Kramer JL, Rowland JH, Stein KD, et al. Cancer treatment and survivorship statistics, 2016. CA Cancer J Clin. 2016;66(4):271-289.

6. Piotrowski G, Gawor R, Stasiak A, Gawor Z, Potemski P, Banach M. Cardiac complications associated with trastu- zumab in the setting of adjuvant chemotherapy for breast cancer overexpressing human epidermal growth factor receptor type 2 - a prospective study. Arch Med Sci. 2012;8(2):227-235.

7. Nabholtz JM, Reese DM, Lindsay MA, Riva A. HER2positive breast cancer: update on Breast Cancer International Research Group trials. Clin Breast Cancer. 2002;3(Suppl 2):S75-79.

8. Fedele C, Riccio G, Malara AE, D'Alessio G, De Lorenzo C. Mechanisms of cardiotoxicity associated with ErbB2 inhibitors. Breast Cancer Res Treat. 2012;134(2):595-602.

9. Keefe DL. Trastuzumab-associated cardiotoxicity. Cancer. 2002;95(7):1592-1600.

10. Seidman A, Hudis C, Pierri MK, Shak S, Paton V, Ashby M, Murphy M, et al. Cardiac dysfunction in the trastuzumab clinical trials experience. J Clin Oncol. 2002;20(5):1215-1221.

11. Genetech: Herceptin (trastuzumab): highlights of prescribing information, 04/2015 update. http://www.gene. com/download/pdf/hercetpin_prescribing.pdf.

12. Perez EA, Romond EH, Suman VJ, Jeong JH, Sledge G, Geyer CE, Jr., Martino S, et al. Trastuzumab plus adjuvant chemotherapy for human epidermal growth factor receptor 2-positive breast cancer: planned joint analysis of overall survival from NSABP B-31 and NCCTG N9831. J Clin Oncol. 2014;32(33):3744-3752.

13. Olin RL, Desai SS, Fox K, Davidson R. Non-myopathic cardiac events in two patients treated with trastuzumab. Breast J. 2007;13(2):211-212.

14. Tu CM, Chu KM, Yang SP, Cheng SM, Wang WB. Trastuzumab (Herceptin)-associated cardiomyopathy presented as new onset of complete left bundle-branch block mimicking acute coronary syndrome: a case report and literature review. Am J Emerg Med. 2009;27(7):903 e901-903.

15. Ribeiro KB, Miranda CH, Andrade JM, Galli LG, Tiezzi DG, Oliveira HF, Zola FE, et al. Trastuzumab-induced myocardiotoxicity mimicking acute coronary syndrome. Case Rep Oncol. 2012;5(1):125-133.

16. Oliveira M, Nave M, Gil N, Passos-Coelho JL. Sudden death during adjuvant trastuzumab therapy of breast cancer. Ann Oncol. 2010;21(4):901.

17. Ferguson C, Clarke J, Herity NA. Ventricular tachycardia associated with trastuzumab. N Engl J Med. 2006;354(6):648-649.

18. Bayar N, Kucukseymen S, Goktas S, Arslan S. Right ventricle failure associated with trastuzumab. Ther Adv Drug Saf. 2015;6(3):98-102. 